\title{
Numerically efficient modeling of CNT transistors with ballistic and non-ballistic effects for circuit simulation
}

\author{
Tom J Kazmierski, Member, IEEE, Dafeng Zhou, Bashir M Al-Hashimi, Fellow, IEEE and Peter Ashburn, \\ Member, IEEE
}

\begin{abstract}
This paper presents an efficient carbon nanotube (CNT) transistor modeling technique which is based on cubic spline approximation of the non-equilibrium mobile charge density. The approximation facilitates the solution of the selfconsistent voltage equation in a carbon nanotube so that calculation of the CNT drain-source current is accelerated by at least two orders of magnitude. A salient feature of the proposed technique is its ability to incorporate both ballistic and nonballistic transport effects without a significant computational cost. The proposed models have been extensively validated against reported CNT ballistic and non-ballistic transport theories and experimental results.
\end{abstract}

Index Terms-Carbon nanotube transistors, numerical modeling, non-ballistic effects, circuit simulation.

\section{INTRODUCTION}

Carbon Nanotube Transistors (CNTs) have been demonstrated to have potential of becoming an attractive solution in addition to their silicon counterparts, mainly due to their electrostatic properties, such as ballistic or near ballistic transport of electrons and very low conduction threshold voltages [1], [2], [3], which make CNTs suitable for ultra high speed and ultra low power circuit design. While physical properties of carbon nanotubes are studied in greater depth and the theory of CNTs becomes better understood, most state-of-theart physical and circuit-level models are currently concerning ballistic or near-ballistic transport [1], [4], [5], [6], [7], [8], [9], [10], [11], [12]. There is a growing need for numerically efficient CNT transistor models suitable for implementation in circuit-level simulators, especially in the light of the recently reported successful implementations of logic circuits built with CNTs [13]. Very recently breakthrough improvements to accurate SPICE-compatible CNT transistor modeling have been proposed where not only the ballistic transport but also a number of non-ballistic effects have been included [11], [12]. However, the main stumbling block in the development of a circuit-level model is the fact that accurate calculation of the mobile charge involves numerical integration of the densities of states over the number of allowed energy levels using the

Tom J Kazmierski, Dafeng Zhou, Bashir M Al-Hashimi and Peter Ashburn are with the School of Electronics and Computer Science, University of Southampton, Southampton, SO17 1BJ, UK (email: tjk,dz05r,bmah,pa@ecs.soton.ac.uk).

Copyright (c) 2009 IEEE. Personal use of this material is permitted. However, permission to use this material for any other other purposes must be obtained from the IEEE by sending a request to pubs-permissions@ieee.org.
Fermi probability distribution. In addition, as the total drain current is affected not only by the non-equilibrium mobile charge in the nanotube but also by the charges present at terminal capacitances, the solution of an implicit non-linear algebraic equation is necessary using some iterative approach, such as the Newton-Raphson method [1], [6]. Resulting CPU times are prohibitive for the purpose of circuit simulation where networks involving large numbers of such devices may need to be analyzed. For example, the MATLAB script named FETToy [14] available on line as a reference implementation of the state-of-the art ballistic CNT theory, requires more than 12 seconds of the CPU time on a Pentium IV PC to calculate a family of current drain characteristics for a single transistor [15]. Inclusion of non-ballistic effects aggravates the problem of excessive CPU time consumption.

It has been recently proposed to eliminate the need for costly Newton-Raphson iterations and the numerical evaluation of the Fermi-Dirac integral while still maintaining a good agreement with the physical theory [8], [16]. These techniques are based on piecewise approximation of the charge density profiles, either linear [8] or non-linear [16] to simplify calculations. In this paper we investigate the use of a cubic spline approximation of the charge density which, like the piecewise nonlinear approximation, also allows a closed-form solution of the self-consistent voltage equation. The main advantage of using cubic splines is an improved control of the approximation accuracy. Furthermore, as some theories on the non-ballistic effects have recently emerged [17], [18], [19], [20], [21], [11], [12], in addition to the ballistic transport model [16] we demonstrate how the proposed approximation can be applied to include non-ballistic behavior.

The approach presented in this paper addresses the need for efficient calculation of the $I_{D S}$ current resulting from ballistic and non-ballistic transport in the carbon nanotube itself. To develop a complete CNT transistor model, a number of additional effects representing non-idealities and parasitics, such as contact effects or series resistances, which are external to the inner CNT transistor, also need to be considered.

\section{MobiLE CHARGE DENSITY AND SELF-CONSISTENT VOLTAGE}

When an electric field is applied between the drain and the source of a CNT transistor illustrated in figure 1, a nonequilibrium mobile charge is induced in the nanotube[1], [22], [23]: 


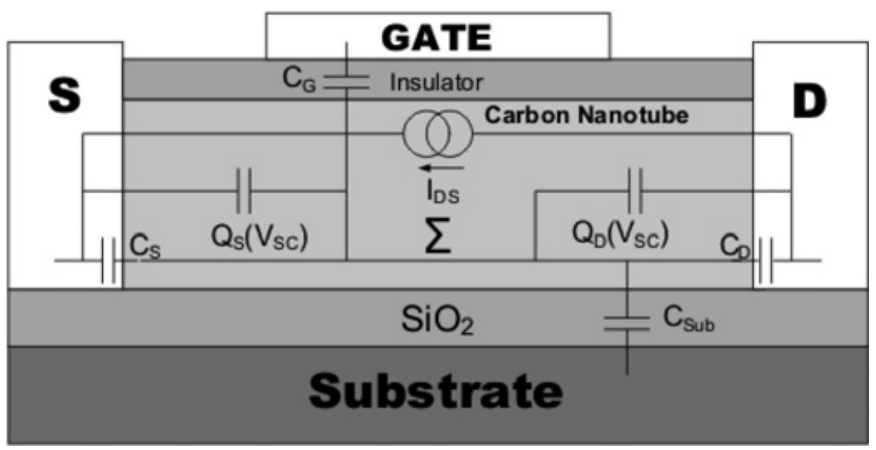

Fig. 1. Structure layout of a top-gate CNT transistor showing components of the proposed equivalent circuit model with the virtual node $\Sigma$ for $V_{S C}$.

$$
\Delta Q=q\left(N_{S}+N_{D}-N_{0}\right)
$$

where $N_{S}$ is the density of positive velocity states filled by the source, $N_{D}$ is the density of negative velocity states filled by the drain and $N_{0}$ is the equilibrium electron density. These densities are determined by the Fermi-Dirac probability distribution as follows:

$$
\begin{aligned}
N_{S} & =\frac{1}{2} \int_{-\infty}^{+\infty} D(E) f\left(E-U_{S F}\right) d E \\
N_{D} & =\frac{1}{2} \int_{-\infty}^{+\infty} D(E) f\left(E-U_{D F}\right) d E \\
N_{0} & =\int_{-\infty}^{+\infty} D(E) f\left(E-E_{F}\right) d E
\end{aligned}
$$

where $D(E), U_{S F}$ and $U_{D F}$ are defined as

$$
\begin{gathered}
D(E)=D_{0} \frac{E}{\sqrt{E^{2}-\left(E_{g} / 2\right)^{2}}} \Theta\left(E-E_{g} / 2\right) \\
U_{S F}=E_{F}-q V_{S C} \\
U_{D F}=E_{F}-q V_{S C}-q V_{D S}
\end{gathered}
$$

$D(E)$ is the density of states at the channel, $D_{0}=$ $8 /\left(3 \pi V_{c c} a_{c c}\right)$ is the constant density of states of a metallic nanotube, $E_{g}$ is the band gap which can be calculated using $2 a_{c c} V_{c c} / d$ [14], where $a_{c c}$ and $V_{c c}$ are the carbon $\pi-\pi$ nearest-neighbor bond length and energy of the tight bonding model respectively. $\Theta\left(E-E_{g} / 2\right)$ equals 1 when $E>E_{g} / 2$ and 0 when $E \leq E_{g} / 2$.

$V_{S C}$ is the self-consistent voltage, a recently introduced concept [1] which illustrates that the CNT energy band is affected by external terminal voltages, $D(E)$ is the density of states, $E_{F}$ is the Fermi level, $f$ is the Fermi probability distribution, $q$ is the electronic charge and $E$ represents the energy levels per nanotube unit length. The self-consistent voltage $V_{S C}$ is implicitly related to the device terminal voltages and charges at terminal capacitances by the following non-linear algebraic equation [1], [8]:

$$
V_{S C}=\frac{-Q_{t}+q N_{S}\left(V_{S C}\right)+q N_{D}\left(V_{S C}\right)-q N_{0}}{C_{\Sigma}}
$$

where $Q_{t}$ represents the charge stored in terminal capacitances and is defined as

$$
Q_{t}=V_{G} C_{G}+V_{D} C_{D}+V_{S} C_{S}+V_{S u b} C_{S u b}
$$

where $C_{G}, C_{D}, C_{S}, C_{S u b}$ are the gate, drain, source and substrate capacitances correspondingly and the total terminal capacitance $C_{\Sigma}$ is

$$
\begin{gathered}
C_{\Sigma}=C_{G}+C_{D}+C_{S}+C_{S u b} \\
C_{o x}=2 \pi k_{1} \varepsilon_{0} / \ln \left(\frac{2 t_{o x}+d}{d}\right) \\
C_{\text {Sub }}=2 \pi k_{2} \varepsilon_{0} / \ln \left(4 H_{\text {Sub }} / d\right)
\end{gathered}
$$

where $d$ is the diameter of the carbon nanotube, $H_{S u b}$ is the thickness of the $\mathrm{SiO}_{2}$ layer on the substrate, $t_{o x}$ is the thickness of the gate insulator and $k_{1}, k_{2}$ are the relative permittivities of the gate and the substrate respectively [24]. Meanwhile, the capacitances between terminals can be obtained as follows as reported previously [14].

$$
\begin{gathered}
C_{G}=C_{o x} \\
C_{S}=0.097 C_{o x} \\
C_{D}=0.040 C_{o x}
\end{gathered}
$$

And the addition of $C_{S u b}$ also implies that the body effects may be taken into account in further work. The standard approach to the solution of equation (8) is to use the NewtonRaphson iterative method and in each iteration evaluate the integrals in equations (3) and (4) to obtain the state densities $N_{D}$ and $N_{S}$.

\section{CIRCUIT MODEL AND SPLINE-BASED APPROXIMATION OF CHARGE DENSITIES}

In an earlier work [15] we proposed to apportion equal parts of the equilibrium mobile charge density $N_{0}$ to the drain and source. This facilitates circuit implementation of the model because now the corresponding non-equilibrium mobile charge densities $Q_{S}$ and $Q_{D}$ can be modeled as non-linear circuit capacitances, dependent on the self-consistent voltage $V_{S C}$, and connected between a conceptual inner node, which represents the self-consistent potential, and CNT terminal nodes:

$$
Q_{S}\left(V_{S C}\right)=q\left(N_{S}\left(V_{S C}\right)-\frac{1}{2} N_{0}\right)
$$

and

$$
Q_{D}\left(V_{S C}\right)=q\left(N_{D}\left(V_{S C}\right)-\frac{1}{2} N_{0}\right)
$$

The resulting equivalent circuit is shown in figure 2 where $\Sigma$ is the hypothetical inner node described above, which 
comprises all the CNT charges. The current $I_{t}$ represents the tunneling, one of the non-ballistic effects discussed in section IV. $I_{D S}$ is the transport current determined by the self-consistent voltage $V_{S C}$. If only ballistic transport is considered, $I_{D S}$ is equivalent to current $I_{D S_{B}}$ given by equation (18) below. In section IV we consider models of non-ballistic effects which allow a more accurate representation of the transport current $I_{D S}$.

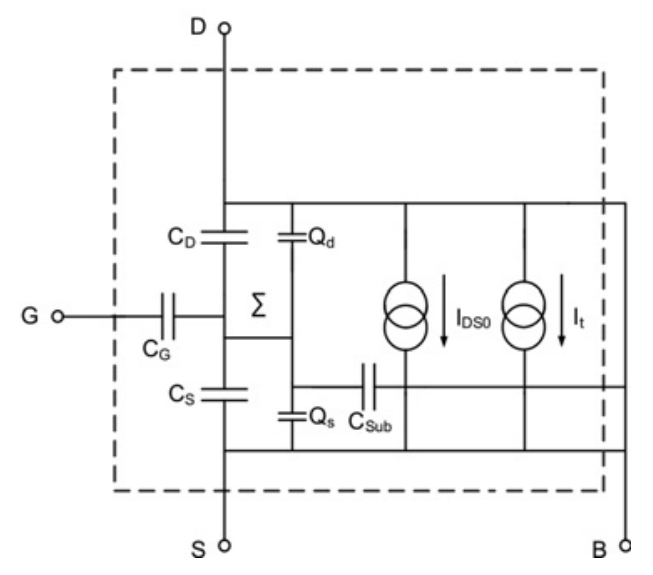

Fig. 2. Equivalent circuit for the proposed CNT transistor model.

According to the ballistic CNT ballistic transport theory [1], [14] the drain current caused by the transport of the nonequilibrium charge across the nanotube can be calculated using the Fermi-Dirac statistics as follows:

$$
I_{D S_{0}}=\frac{2 q k T}{\pi \hbar}\left[\mathcal{F}_{0}\left(\frac{U_{S F}}{k T}\right)-\mathcal{F}_{0}\left(\frac{U_{D F}}{k T}\right)\right]
$$

where $\mathcal{F}_{0}$ represents the Fermi-Dirac integral of order $0, k$ is Boltzmann's constant, $T$ is the temperature and $\hbar$ is reduced Planck's constant.

If the self-consistent voltage $V_{S C}$ is known, the evaluation of the drain current poses no numerical difficulty as energy levels $U_{S F}, U_{D F}$ can be found quickly from equations 6,7 and $I_{D S}$ calculated directly using the closed-form analytical solution of the Fermi-Dirac integral of order 0 [25]. However, as it has already been highlighted above, the solution of the self-consistent voltage equation (8) is very time consuming because it involves a Newton-Raphson iterative process in which each iteration requires numerical integration to obtain state densities $N_{D}\left(V_{S C}\right)$ and $N_{D}\left(V_{S C}\right)$. This is the main drawback of general methods in calculating charge densities. The next section outlines a piecewise approximation technique that eliminates the need for these complex calculations while maintaining a high modeling accuracy.

Our earlier work [16] proposed a piecewise non-linear approximation technique that eliminates the need for these complex calculations. Although the piecewise non-linear approach was demonstrated to be very fast and accurate in the transistor modeling, it requires a complex fitting process when deciding on the number of approximation pieces and intervals of the ranges, which makes this type of modeling inflexible. To improve the ease of use of the model without losing much of the computational efficiency, a piecewise approximation of the mobile charge based on cubic splines [26] can be used as an alternative. While cubic splines are generally cumbersome to apply in semiconductor modeling where multi-dimensional approximations are usually required, here they are particularly suitable and easy to apply because the dependence of nonequilibrium mobile charge on the self-consistence voltage is one dimensional. As illustrated below, the numerical efficiency of the ballistic CNT transport model hinges on an efficient calculation of the non-equilibrium mobile charge. For the charge density defined in Section II

$$
Q\left(V_{S C}\right)=\frac{q}{2} \int_{-\infty}^{+\infty} D(E) f\left(E-E_{F}-V_{S C}\right) d E
$$

a simple spline structure can be build, using $n$ equally spaced points, to approximate the mobile charge dependence on $V_{S C}$ using cubic polynomial pieces of the following form

$$
Q\left(V_{S C}\right)=a_{i} V_{S C}^{3}+b_{i} V_{S C}^{2}+c_{i} V_{S C}+d_{i}
$$

where $a_{i}, b_{i}, c_{i}$ and $d_{i}, i=1, \ldots, n-1$ are spline coefficients. This enables a closed-form solution of the selfconsistent voltage equation (8) as, for each piece, it now becomes a a polynomial equation of the third order. Thus, the need for costly Newton-Raphson iterations and evaluations of Fermi-Dirac integrals is eliminated.

\section{NON-BALLISTIC TRANSPORT EFFECTS}

Research into non-ballistic transport in carbon nanotubes has recently yielded results and some new theories have been reported. Studies of the energy domain reveal that the incommensurate system within a non-ideal nanotube implies the existence of a general non-ballistic regime [17]. The transport type in carbon nanotubes, ballistic or non-ballistic, depends on the energy region. Studies of the energy domain reveal that the incommensurate system within a non-ideal nanotube implies the existence of a general non-ballistic regime [17]. For a CNT transistor with the length smaller than the carrier mean free path but larger than the Coulomb blockade length, the ballistic transport will dominate. To travel through a single defect Coulomb potential, the transmission coefficient can be calculated by $T_{\text {defect }}(E)=G_{\text {filled }}(E) / G_{\text {empty }}(E)$ [18], where $E$ is the energy of the hole in a nanotube. Therefore, the charging and discharging decides the maximum differential conductance through single transport channels, and $\mathrm{E}$ is directly determined by the terminal voltage $V_{D S}$. For nonballistic CNTs, this transmission coefficient fluctuation could be caused by mobility fluctuation. However, under the effects of scattering, $E$ is much smaller than $\mathrm{q} V_{D S}$ in the case. It has also been shown that a mismatch of helicity between adjacent shells may result in a short mean-free path [19]. In addition, all kinds of likely defects, such as vacancies, contamination, contact to the substrate, and adsorbed molecules may cause non-ballistic transport [19]. In the light of these results, the non-ballistic transport in CNTs is likely to attract more research attention in the near future. Below we outline four major non-ballistic effects which have been implemented in our numerical models. 


\section{A. Elastic scattering}

The elastic scattering mechanism in the CNT channel region affects the channel resistance and therefore makes a potential drop of the channel voltage. Assuming that mean free path (MFP) $l_{e f f}$ is proportional to the diameter of the nanotube [27], [28], which is $l_{\text {eff }}=d /\left(d_{0}\right) \lambda_{\text {eff }}$, where $d_{0}$ is the reference diameter when $\lambda_{e f f}$ is the elastic-scattering MFP [12], and the transmission probability in the elastic-scattering channel region can be expressed using $T_{\text {eff }}=l_{\text {eff }} /\left(L+l_{\text {eff }}\right)$, where $d$ is the CNT diameter, $\lambda_{\text {eff }} \approx 200 \mathrm{~nm}$ [29] and $L$ is the channel length. The channel potential drop can hereby be derived as

$$
V_{D S e f f}=\frac{L}{L+\frac{d}{d_{0}} \cdot \lambda_{e f f}} V_{D S}
$$

The contribution to the device current characteristics can be computed directly from the variable voltage $V_{D S e f f}$ due to the elastic scattering instead of the channel resistance, which simplifies the calculation efficiently [12]. Figure 3 illustrates how the drain current changes when the elastic scattering effect relating to the channel length is considered.

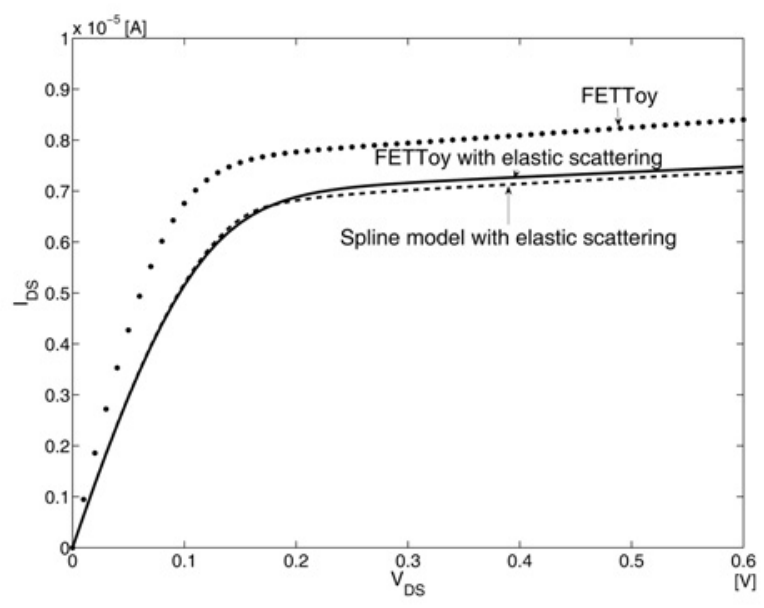

Fig. 3. Comparison of the drain currents at $V_{G}=0.6 \mathrm{~V}, \mathrm{~T}=300 \mathrm{~K}$, $E_{F}=-0.32 \mathrm{eV}$ and $L=300 \mathrm{~nm}$ for models with the elastic scattering effect: FETToy model (dotted line), the FETToy-plus model (solid line) and the proposed spline Model 1 with the elastic scattering effect (dashed line).

\section{B. Band gap tuning with strain}

It has been demonstrated that the transport property of a CNT can vary under strain [30]. Measurements have shown that the strain exerted onto a nanotube can change the band gap thus affect the transport characteristics. The shape distortion formed by the strain can be treated as a key factor when calculating the extra band gap caused by the effect.

$$
E_{\text {geff }}=E_{g}+\frac{d E_{\text {gstrain }}}{d \chi} \chi
$$

where $E_{g e f f}$ is the effective band gap under strain, $d E_{\text {gstrain }}$ is the gap shift due to the strain and $\chi$ is defined as the distortion factor under strain.
It has also be indicated that the change rate of the band gap $\frac{d E_{\text {gstrain }}}{d \chi}$ in the light of strain is chirality dependent, which can be computed using

$$
\frac{d E_{\text {gstrain }}}{d \chi}=3 \sigma\left(1+r_{0}\right) \operatorname{sign}(2 p+1) \cos (3 \phi)
$$

In the equation, $\sigma$ is the overlap integral of the tight-binding $\mathrm{C}-\mathrm{C}$ model, with a value of circa $2.7 \mathrm{eV}, r_{0} \approx 0.2$ is the Poisson's ratio, $\phi$ is the chiral angle of the nanotube and $p$ comes from the CNT chirality: for a CNT with the chirality $(m, n), m-n=3 l+p$, where $l$ and $p$ are both integrals. It is indicated that the chirality and strain can both influence the band gap of a CNT and the total gap $E_{g e f f}$ could be either larger or less than the ideal diameter-based calculation $E_{g}$, which might cause the transport to decrease or increase respectively.

Figure 4 illustrates that when under certain strain conditions $\left(\chi=0.1, p=1\right.$ and $\left.\phi=20^{\circ}\right)$ the drain current has been reduced due to the bang gap variation.

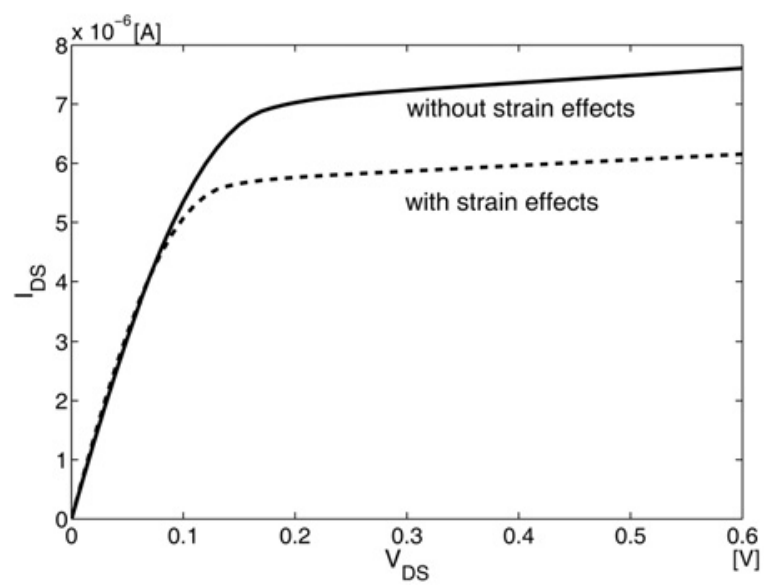

Fig. 4. Comparison of the drain currents at $V_{G}=0.6 \mathrm{~V}$ for models with (dashed line) and without (solid line) strain effects for a $300 \mathrm{~nm}$ long CNT channel with the diameter of $1 \mathrm{~nm}$ at $E_{F}=-0.32 \mathrm{eV}$ and $T=300 \mathrm{~K}$.

\section{Tunneling effect}

The tunneling effect is also inevitable in the subthreshold region, which may cause self-consistence potential lowering and thereby worsen the threshold characteristics of the transistor. One simplified method to describe the tunneling effect is to introduce a parameter $T_{t}$, called the tunneling probability [11], which is calculated as

$$
T_{t} \approx \frac{\pi^{2}}{9} e^{-\frac{\pi \sqrt{m^{*} E_{g}^{3}}}{\sqrt{8} q \hbar F}}
$$

where $F$ is a parameter which triggers the tunneling under high electrical field [11] and $m^{*}$ is the effective electron mass [11]. The tunneling current then can be obtained by $T_{t}$ timing the maximum possible tunneling current using 


$$
\begin{gathered}
I_{t}=\frac{4 q k T}{h} T_{t} \sum_{m=1}^{M}\left[\ln \left(1+e^{\left(q V_{D S e f f}-E_{g e f f} / 2-E_{F}\right) / K_{B} T}\right)\right. \\
\left.-\ln \left(1+e^{\left(q V_{D S e f f}-E_{F}\right) / K_{B} T}\right)\right] \frac{\max \left(q V_{D S e f f}-E_{g e f f}, 0\right)}{q V_{D S e f f}-E_{g e f f}}
\end{gathered}
$$

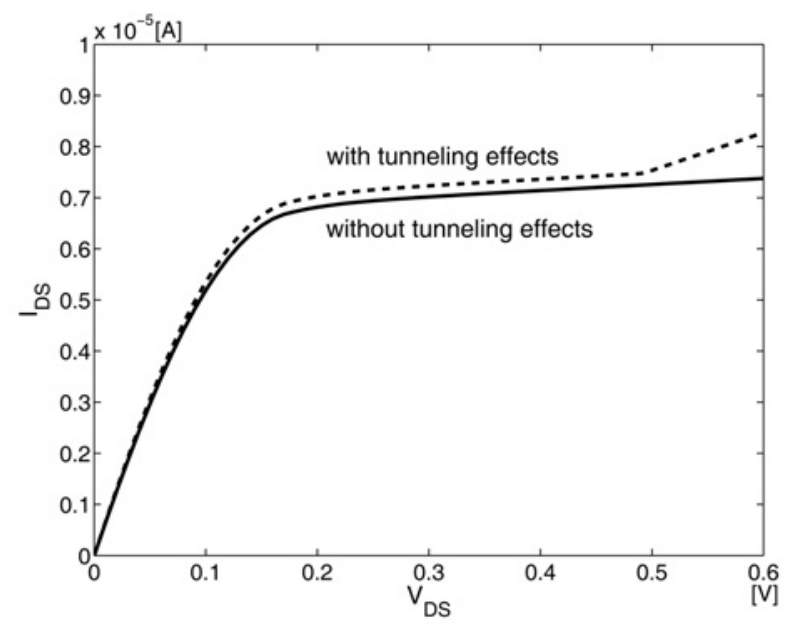

Fig. 5. Comparison of the drain currents at $V_{G}=0.6 \mathrm{~V}$ for models with (circlet line) and without (solid line) tunneling effects for a $300 \mathrm{~nm}$ long CNT channel with the diameter of $1 \mathrm{~nm}$ at $E_{F}=-0.32 \mathrm{eV}$ and $T=300 \mathrm{~K}$.

It can be noticed from figure 5 that the tunneling current has increased the total drain current throughout the $V_{D S}$ range, but the effect only becomes obvious when the bias voltage is getting large and exceed a certain turning point depending on the coefficients.

\section{Phonon scattering}

For semiconducting carbon nanotubes, the scattering effects are related to the band energy. The effective phonon scattering mean free path in a semiconducting nanotube can be computed by

$$
\begin{gathered}
\frac{1}{l_{s c}\left(V_{x}\right)}=\frac{1}{l_{a p}}\left[1-\frac{1}{1+e^{\left(E_{F}-q V_{S C}+q V_{x}\right) / K_{B} T}}\right] \\
+\frac{1}{l_{o p}}\left[1-\frac{1}{1+e^{\left(E_{F}-q V_{S C}-\hbar \omega_{o p}+q V_{x}\right) / K_{B} T}}\right]
\end{gathered}
$$

where $l_{a p}=500 \mathrm{~nm}$ is a typical acoustic phonon scattering MFP value while $l_{o p}=15 \mathrm{~nm}$ is a typical optical phonon scattering MFP, and $\hbar \omega_{o p} \approx 0.16 \mathrm{eV}$ is a typical OP energy [20], [21]. It can be noticed that at low carrier energy (e.g. < $0.15 \mathrm{eV}$ ), the acoustic scattering dominates; while the optical scattering is more important at high kinetic energy.

$$
\begin{gathered}
T_{S}=\frac{l_{s c}(0)}{l_{s c}(0)+L} \\
T_{D}=\frac{l_{s c}\left(V_{D S e f f}\right)}{l_{s c}\left(V_{D S e f f}\right)+L}
\end{gathered}
$$

$$
\begin{array}{r}
I_{D S_{p}}=\frac{2 q k T}{\pi \hbar}\left[T_{S} \ln \left(1+e^{\frac{E_{F}-q V_{S C}}{k T}}\right)\right. \\
\left.-T_{D} \ln \left(1+e^{\frac{E_{F}-q\left(V_{S C}+V_{D S e f f}\right)}{k T}}\right)\right]
\end{array}
$$

Equations (27),(28) and (29) are to describe the scattering effects on the $I-V$ characteristics. Figure 6 illustrates that the phonon scattering effects may limit the transport capability of carriers in the channel and hence restrain the drain current. Different from equation (18), the scattering coefficients $T_{S}$ and $T_{D}$ are introduced in equation (29) which indicates the effects of the phonon scattering.

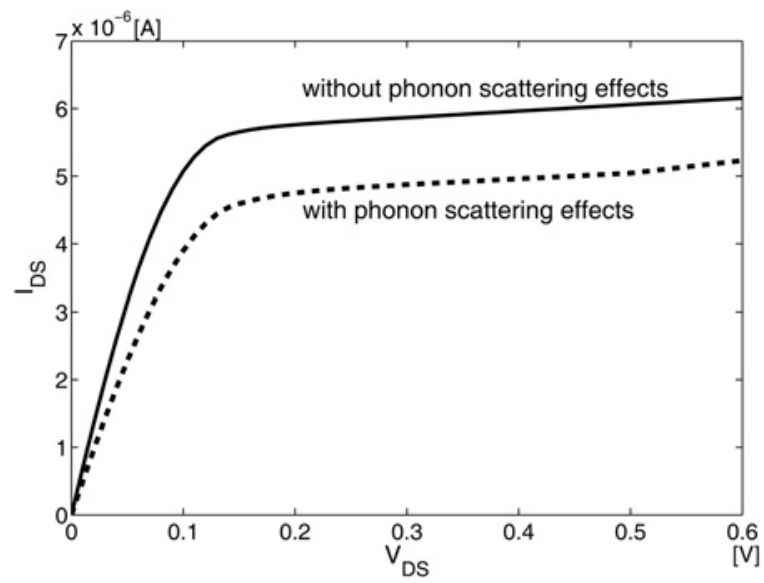

Fig. 6. Comparison of the drain currents at $V_{G}=0.6 \mathrm{~V}$ for models with (dashed line) and without (solid line) phonon scattering effects for a $300 \mathrm{~nm}$ long CNT channel with the diameter of $1 \mathrm{~nm}$ at $E_{F}=-0.32 \mathrm{eV}$ and $T=$ $300 K$.

It can be seen from above subsections that some non-ideal effects exist when operating a CNT transistor and therefore the transport characteristics becomes non-ballistic. There can be quite a large number of factors including scattering mechanisms, parasitic capacitances and energy barriers as well, and the transport phenomenons introduced above are just part of them. Some non-idealities, such as contact junction effects, are also of great importance. However, more theoretical analysis and mathematic expressions are needed before they can be added into the model. In reality, the non-ballistic effects are caused by a number of factors, including fabrication technologies and materials, etc. Therefore, it becomes difficult to identify which effect is more important, and it can be seen certain effects are dominant when the related coefficients are of great value in the provided device. For a model with nonballistic effects introduces above, the transport equation can be treated as the summary of the transport current and the tunneling current, which is also reflected in figure 2 as the parallel current sources.

By adding equations (21), (22), (25) and (29) to the original FETToy MATLAB scripts, we developed an extended model named FETToy + [31] which has included the above four nonballistic effects in the model. Figure 7 shows an accuracy comparison between FETToy and FETToy+ which combines the effects shown in Figures 3, 4, 5 and 6 respectively. 


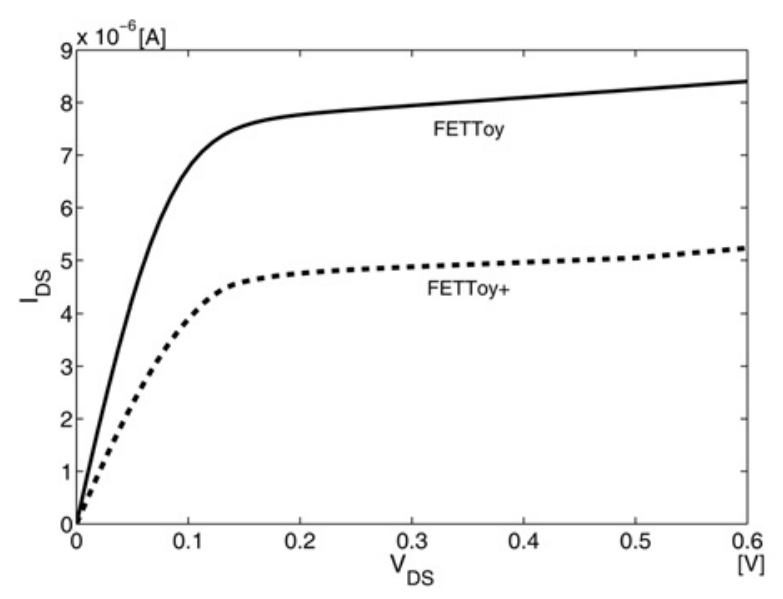

Fig. 7. Comparison of the drain currents at $V_{G}=0.6 \mathrm{~V}$ for FETToy (solid line) and FETToy+ (dashed line) models with $300 \mathrm{~nm}$ channel length and $1 \mathrm{~nm}$ diameter at $E_{F}=-0.32 \mathrm{eV}$ and $T=300 \mathrm{~K}$.

\section{Performance of Proposed Model}

For an ideal model with no non-ballistic effects, once the self-consistent voltage $V_{S C}$ is efficiently calculated from the closed-form solutions of equation (8) after the approximation which yields only linear, quadratic or a 3 rd order polynomial relations, the total drain current can be directly obtained from equations (6), (7) and (18). However, when non-ballistic transport features are considered, a more complicated model which includes additional coefficients and equations is needed. To clarify the distinction and compare the performance of the proposed models, separate simulations have been carried out for both ideal and non-ballistic scenarios.

\section{A. Ballistic model}

In the ballistic modeling approach, the calculations are extremely fast, as Newton-Raphson iterations and integration of the Fermi-Dirac probability distribution are now eliminated. Table I shows the average CPU times for proposed models and those for FETToy. For accurate measurement, experiments were carried out by invoking all models 100 times. Results show that spline models are more than two orders of magnitude faster than FETToy. The extent to which the modeling accuracy was compromised by numerical approximation was also measured by calculating average RMS errors in the simulations. Here we chose the concept of normalized RMS error which is expressed in equation (30).

$$
\text { normalized } R M S E=\frac{\sqrt{\frac{\sum_{i=1}^{n}\left(a_{i}-b_{i}\right)^{2}}{n}}}{\max \left(a_{i}, b_{i}\right)-\min \left(a_{i}, b_{i}\right)}
$$

Figures 8 and 9 show the $I_{D S}$ characteristics calculated by FETToy compared with two spline ballistic models, Model 1 and Model 2 using $n=4$ and $n=5$ points respectively. As shown in Table I, both models maintain a high accuracy in terms of the average RMS error. As expected, Model 2 is slightly more accurate with errors not exceeding $1.1 \%$ at $T=300 \mathrm{~K}$ and $E_{F}=-0.32 \mathrm{eV}$ throughout the typical ranges of drain voltages $V_{D S}$ and gate bias $V_{G}$.

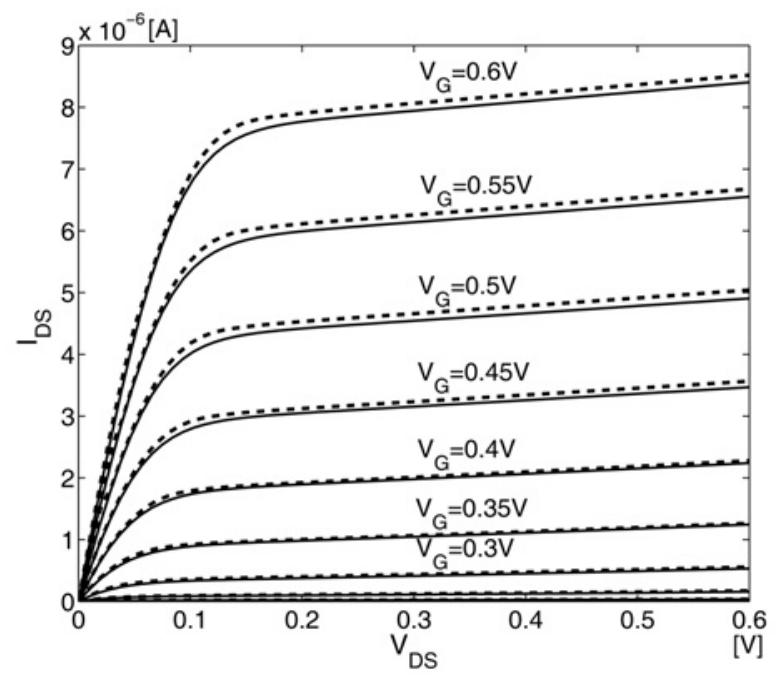

Fig. 8. Drain current characteristics at $T=300 \mathrm{~K}$ and $E_{F}=-0.32 \mathrm{eV}$ for FETToy(solid lines) and piecewise approximation using ballistic Model 1 (dashed lines).

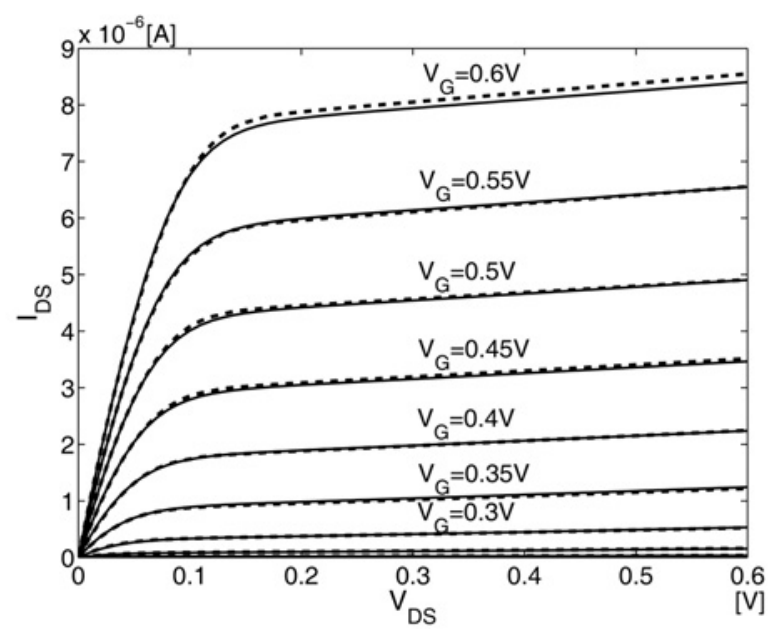

Fig. 9. Drain current characteristics at $T=300 \mathrm{~K}$ and $E_{F}=-0.32 \mathrm{eV}$ for FETToy(solid lines) and piecewise approximation using ballistic Model 2 (dashed lines).

TABLE I

COMParison OF AVERAge CPU TIME AND MAXIMUM RMS ERror of PROPOSED BALLISTIC MODELS WITH FETTOY.

\begin{tabular}{c|c|c|c}
\hline $\begin{array}{c}\text { FETToy } \\
\text { CPU time }\end{array}$ & $\begin{array}{c}\text { Spline } \\
\text { model }\end{array}$ & $\begin{array}{c}\text { Max } \\
\text { RMSE }\end{array}$ & $\begin{array}{c}\text { CPU } \\
\text { time }\end{array}$ \\
\hline \multirow{2}{*}{$1287 \mathrm{sec}$} & Model 1 & $1.4 \%$ & $11.7 \mathrm{Sec}$ \\
\cline { 2 - 4 } & Model 2 & $1.1 \%$ & $19.3 \mathrm{Sec}$ \\
\hline
\end{tabular}

\section{B. Models with ballistic and non-ballistic effects}

When taking into account the non-ballistic effects described in section IV, the FETToy model [14], which implements the purely ballistic transport theory, cannot be used as a reference for accuracy and speed analysis. By adding equations (21), (22), (25) and (29) to the original FETToy MATLAB scripts, we developed an extended model named FETToy+ [31] which is capable of analyzing both ballistic and non- 
ballistic performance of CNTs. Similarly, the cubic spline model has been enhanced to include the non-ideal effects. The second group of simulations illustrate that the drain current may be reduced dramatically due to the presence of nonballistic effects. We have tested two spline-based non-ballistic models, Model 3 and Model 4 with $n=4$ and $n=5$ spline points correspondingly. Figure 10 and 11 show the simulation results for FETToy+ and both the spline non-ballistic models. In addition, the accuracy and the speed of the proposed models have been measured and compared with those of FETToy+, and results are listed in Table II. It can also be seen that the non-ballistic models consume more CPU time than ballistic models due to more complicated calculations.

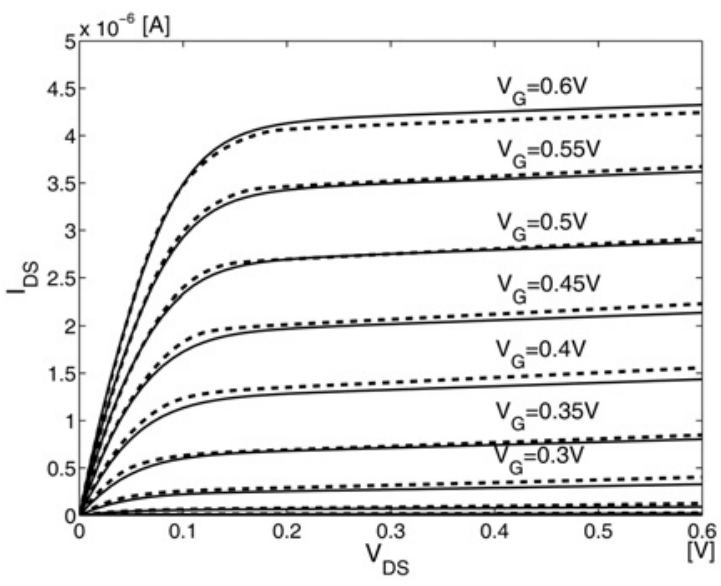

Fig. 10. Drain current characteristics at $T=300 K$ and $E_{F}=$ $-0.32 \mathrm{eV}$ for a $L=300 \mathrm{~nm} \mathrm{CNT}$ channel including non-ballistic effects for FETToy+(solid lines) and piecewise approximation using non-ballistic Model 3 (dashed lines).

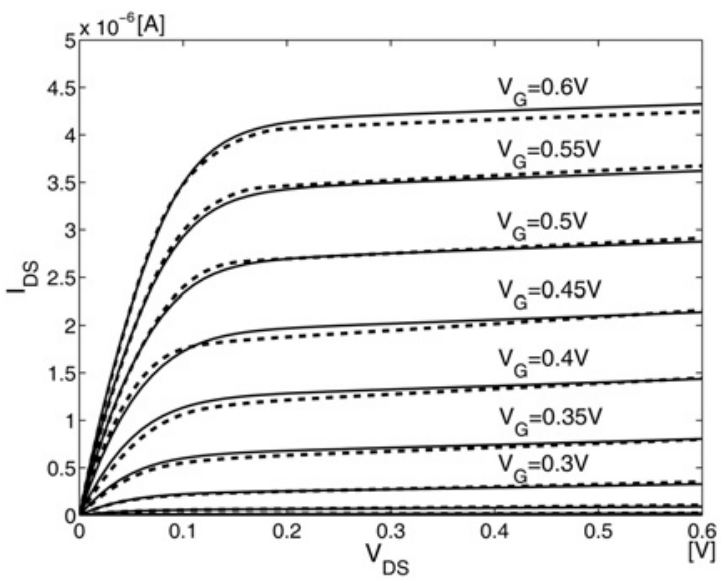

Fig. 11. Drain current characteristics at $T=300 K$ and $E_{F}=$ $-0.32 \mathrm{eV}$ for a $L=300 \mathrm{~nm} \mathrm{CNT}$ channel including non-ballistic effects for FETToy+(solid lines) and piecewise approximation using non-ballistic Model 4 (dashed lines)

\section{COMPARISON WITH EXPERIMENTAL RESULTS}

Additionally, to validate the performance of the proposed models, some reported experimental characteristics were compared with the simulation results for $d=1.6 n m, t_{o x}=$
TABLE II

MAXIMUM RMS ERror to FETTOY+ AND AVERAge CPU TIMES OF SPLINE Models With NON-BALLISTIC EFFECTS.

\begin{tabular}{c|c|c|c}
\hline $\begin{array}{c}\text { FETToy+ } \\
\text { CPU time }\end{array}$ & $\begin{array}{c}\text { Spline } \\
\text { model }\end{array}$ & $\begin{array}{c}\text { Max } \\
\text { RMSE }\end{array}$ & $\begin{array}{c}\text { CPU } \\
\text { time }\end{array}$ \\
\hline $2261 \mathrm{sec}$ & Model 3 & $1.9 \%$ & $13.4 \mathrm{sec}$ \\
\cline { 2 - 4 } & Model 4 & $1.5 \%$ & $22.8 \mathrm{Sec}$ \\
\hline
\end{tabular}

TABLE III

AVERAGE RMS ERRORS IN $I_{D S}$ COMPARISON TO THE EXPERIMENTAL RESULTS OF FETTOY MODEL AND THE PROPOSED NON-BALLISTIC (NB) MODELS FOR $d=1.6 \mathrm{~nm}, t_{o x}=50 \mathrm{~nm}, T=300 \mathrm{~K}$ AND $E_{F}=-0.05 \mathrm{eV}$.

\begin{tabular}{l|c|c}
\hline$V_{G}[V]$ & Spline Model 3 & Spline Model 4 \\
\hline 0.2 & $13.3 \%$ & $12.8 \%$ \\
\hline 0.4 & $12.5 \%$ & $11.9 \%$ \\
\hline 0.6 & $11.3 \%$ & $10.6 \%$ \\
\hline
\end{tabular}

$50 \mathrm{~nm}, T=300 \mathrm{~K}$ and $E_{F}=-0.05 \mathrm{eV}$. Figures 12 shows that the proposed cubic spline model with non-idealities obtains close drain current performance to the experimental measurements which were derived recently and provided sufficient information about the transistor parameters [32]. The n-type carbon nanotube transistor was fabricated with K-doping and grounded back gate in the reported experiment. Table III shows the corresponding average normalized RMS errors. As it can be seen from the following table and figure, both models maintain high accuracy over a wide range temperature and Fermi level values for different carbon-nanotube diameters with the non-ballistic Model 4 being slightly mode accurate.

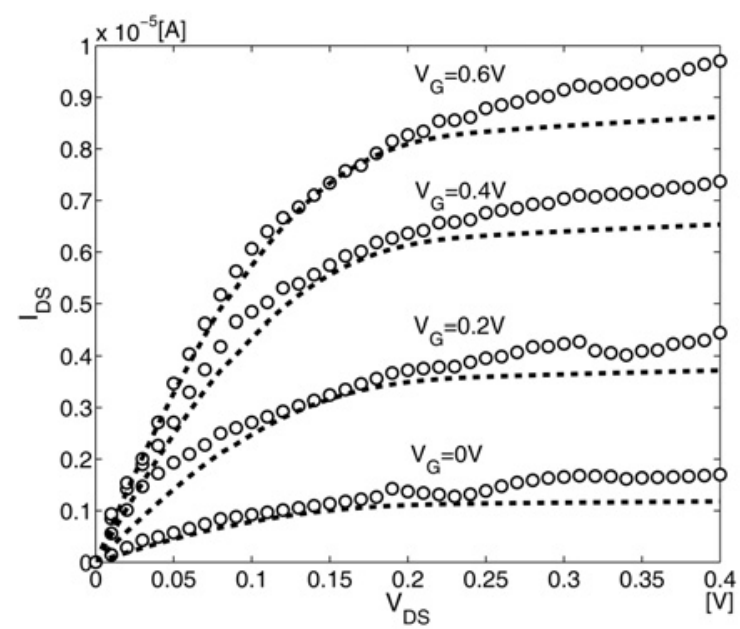

Fig. 12. Comparison to the experimental results(circlet lines) of the proposed non-ballistic Model 4 (dashed lines) for $d=1.6 \mathrm{~nm}, t_{o x}=50 \mathrm{~nm}, T=$ $300 \mathrm{~K}$ and $E_{F}=-0.05 \mathrm{eV}$.

\section{CONCLUSION}

A fast and efficient numerical approach to CNT transistor modeling has been proposed. It allows a straightforward incorporation of both ballistic and non-ballistic transport effects. 
Results have shown that CPU times can be accelerated by two, or in some cases three, orders of magnitude compared with the traditional approach where time-consuming FermiDirac integral and Newton-Raphson iterations are used. The key advantage of the presented technique is that it overcomes numerical difficulties in the calculation of the drain-source current by allowing a closed-form solution of the self-consistent voltage equation. Numerical integration and Newton-Raphson iterations are therefore avoided leading to a substantial acceleration in the model evaluation. The presented model concerns ballistic and non-ballistic transport in the carbon nanotube itself. Future work involves the development of an enhanced CNT transistor model by adding effects external to the inner CNT transistor, such as the Schottky barrier between the carbon nanotube and metal contacts, multiple CNTs at a single gate, channel fringe capacitances, parasitic source/drain resistance, series resistance due to the scattering effects.

\section{ACKNOWLEDGMENT}

The authors would like to acknowledge the support of EPSRC/UK for funding this project in part under grant $\mathrm{EP} / \mathrm{E} 035965 / 1$. We are also grateful to the reviewers for their detailed and constructive comments on the manuscript which helped to improve the paper.

\section{REFERENCES}

[1] Anisur Rahman, Jing Guo, Supriyo Datta, and Mark S. Lundstrom. Theory of ballistic nanotransistors. Electron Devices, IEEE, 50(9):18531864, September 2003.

[2] Phaedon Avouris, Joerg Appenzeller, Richard Martel, and Shalom J. Wind. Carbon nanotube electronics. Proceedings of the IEEE, 91(11):1772-84, November 2003.

[3] Arijit Raychodhury and Kaushik Roy. Carbon nanotube electronics: design of high-performance and low-power digital circuits. IEEE Transactions on Circuits and Systems - I: Fundamental Theory and Applications, 54(11):2391-2401, November 2007.

[4] Arijit Raychowdhury, Saibal Mukhopadhyay, and Kaushik Roy. A circuit-compatible model of ballistic carbon nanotube field-effect transistors. Applied Physics Letters, 23(10):1411-20, October 2004.

[5] Bipul C. Paul, Shinobu Fujita, Masaki Okajima, and Thomas Lee. Modeling and analysis of circuit performance of ballistic CNFET. In 2006 Design Automation Conference, San Francisco, CA, USA, 24-28 July 2006.

[6] Jie Deng and H.-S.Philip Wong. A circuit-compatible SPICE model for enhancement mode carbon nanotube field effect transistors. In 2006 International Conference on Simulation of Semiconductor Processes and Devices, Piscataway, NJ, USA, 6-8 September 2006.

[7] Kenji Natori, Yoji Kimura, and Tomo Shimizu. Characteristics of a carbon nanotube field-effect transistor analyzed as a ballistic nanowire field-effect transistor. Journal of Applied Physics, 97(3):034306-1034306-7, February 2005.

[8] Hamidreza Hashempour and Fabrizio Lombardi. An efficient and symbolic model for charge densities in ballistic carbon nanotube FETs. IEEE-NANO, 1:17-20, 2006.

[9] S. Hasan, S. Salahuddin, M. Vaidyanathan, and M.A. Alam. Highfrequency performance projections for ballistic carbon-nanotube transistors. Nanotechnology, IEEE, 5(1):14-22, January 2006.

[10] Francois Leonard and Derek A Stewart. Properties of short channel ballistic carbon nanotube transistors with ohmic contacts. Nanotechnology, IEEE, 17(18):4699-4705, September 2006.

[11] Jie Deng and H.-S.Philip Wong. A compact spice model for carbonnanotube field-effect transistors including nonidealities and its application - part i: model of the intrinsic channel region. IEEE Transactions on Electron Devices, 54:3186-3194, 2007.

[12] Jie Deng and H.-S.Philip Wong. A compact spice model for carbonnanotube field-effect transistors including nonidealities and its application - part ii: full device model and circuit performance benchmarking. IEEE Transactions on Electron Devices, 54:3195-3205, 2007.
[13] Zhihong Chen, Joerg Appenzeller, Yu-Ming Lin, Jennifer Sippel-Oakley, Andrew G. Rinzler, Jinyao Tang, Shalom J. Wind, Paul M. Solomon, and Phaedon Avouris. An integrated logic circuit assembled on a single carbon nanotube. Science, 311(5768):1735, March 2006.

[14] Anisur Rahman, Jing Wang, Jing Guo, Sayed Hasan, Yang Liu, Akira Matsudaira, Shaikh S. Ahmed, Supriyo Datta, and Mark Lundstrom. Fettoy 2.0 - on line tool, 14 February 2006. https://www.nanohub.org/ resources/220/.

[15] Tom J. Kazmierski, Dafeng Zhou, and Bashir M. Al-Hashimi. A fast, numerical circuit-level model of carbon nanotube transistor. In IEEE Int. Workshop on Design and Test of Defect-Tolerant Nanoscale Architectures (Nanoarch), Santa Clara, CA, 21-22 October 2007.

[16] Tom J. Kazmierski, Dafeng Zhou, and Bashir M. Al-Hashimi. Efficient circuit-level modelling of ballistic cnt using piecewise non-linear approximation of mobile charge density. In IEEE Int. conf. on Design, Automation and Test in Europe (DATE), Munich, Germany, 10-14 March 2008.

[17] Jean-Christophe Charlier, Xavier Blase, and Stephan Roche. Electronic and transport properties of nanotubes. Reviews of Modern Physics, 79(2):677-732, 2007.

[18] Fei Liu, Kang L. Wang, Chao Li, and Chongwu Zhou. Study of random telegraph signals in single-walled carbon nanotube field effect transistors. IEEE Transactions on Nanotechnology, 5(5):441-445, September 2006.

[19] Akinobu Kanda, Kazuhito Tsukagoshi, Yoshinobu Aoyagi, and Youiti Ootuka. Gate-voltage dependence of zero-bias anomalies in multiwall carbon nanotubes. Phys Rev Lett, 92(3):036801, 2004.

[20] Jing Guo and Mark S. Lundstrom. Role of phonon scattering in carbon nanotube field-effect transistors. Applied Physics Letters, 86:193103-13, 2005.

[21] Asha Balijepalli, Saurabh Sinha, and Yu Cao. Compact modeling of carbon nanotube transistor for early stage process-design exploration. In ISLPED'07: 2007 International Symposium on Low Power Electronics and Design, Portland, OR, United States, 27-29 August 2007.

[22] Ming-Hsun Yang, Kenneth B. K. Teo, Laurent Gangloff, William I. Milne, David G. Hasko, Yves Robert, and Pierre Legagneux. Advantages of top-gate, high-k dielectric carbon nanotube field-effect transistors. Applied Physics Letters, 88(11):113507-1-3, March 2006.

[23] Paul L. McEuen, Michael S. Fuhrer, and Hongkun Park. Single-walled carbon nanotube electronics. Nanotechnology, IEEE Transactions, 1(1):78-845, March 2002.

[24] Jie Deng and H.-S.Philip Wong. Modeling and analysis of planar-gate electrostatic capacitance of 1 -d fet with multiple cylindrical conducting channels. IEEE Transactions on Electron Devices, 54:2377-2385, 2007.

[25] Ashley H. Carter. Classical and Statistical Thermodynamics. Benjamin Cummings, May 2000.

[26] John H. Mathews. Numerical analysis - numerical methods, 2005. http: //math.fullerton.edu/mathews/numerical.html.

[27] Carter T. White and Tchavdar N. Todorov. Carbon nanotubes as long ballistic conductors. Nature, 393:240-242, 1998.

[28] Jie Jiang, Jinming Dong, H.T Yang, and D.Y Xing. Universal expression for localization length in metallic carbon nanotubes. Physical Review $B, 64: 045409 / 1-4,2001$.

[29] Islamshah Amlani, Jonathan Lewis, King Lee, Ruth Zhang, Jie Deng, and H.-S. Philip Wong. First demonstration of ac gain from a singlewalled carbon nanotube common-source amplifier. In Electron Devices Meeting, 2006. IEDM '06. International, pages 559-562, 11-13 December 2006.

[30] E. D. Minot, Yuval Yaish, Vera Sazonova, Ji-Yong Park, Markus Brink, and Paul L. McEuen. Tuning carbon nanotube band gaps with strain. Physical Review Letters, 90(15):156401/1-4, April 2003.

[31] Dafeng Zhou, Tom J Kazmierski, Bashir M Al-Hashimi, and Peter Ashburn. Southampton CNT resources, 6 August 2008. https://www. cnt.ecs.soton.ac.uk.

[32] Ali Javey, Ryan Tu, Damon Farmer, Jing Guo, Roy Gordon, and Hongjie Dai1. High performance n-type carbon nanotube field-effect transistors with chemically doped contacts. Nano Letters, 5:345-348, 2005. 


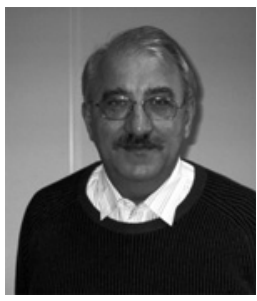

Tom Kazmierski (M'95) received his M.Sc. degree in Electronic Engineering in 1973 from the Warsaw University of Technology and the Ph.D. degree in 1976 from the Military Academy of Technology in Warsaw.

In 1984 he joined the Department of Electronics and Computer Science at the University of Southampton in the U.K., where he pursues research into numerical modeling, simulation and synthesis techniques for computer-aided design of VLSI circuits. In 1990-91 he worked as a Visiting Research Scientist at IBM VLSI Technology Division in San Jose where he developed and patented synchronization techniques for multi-solver simulation backplanes. He has published over 100 papers and given a number of invited talks and tutorials mostly in the area of analogue and mixed-signal synthesis and hardware description languages. Tom Kazmierski has contributed to the development of the VHDL-AMS standard by IEEE, served as Chair of the IEEE DASC P1076.1 (VHDL-AMS) Working Group from 1999 until 2005 and is currently serving as the P1076.1 WG Secretary.

In recent years he has been working on web-based electronic design frameworks and applications of VHDL-AMS to high-level system modeling and synthesis, involving modeling of mixed-domain systems, automated analogue and mixed-signal synthesis for ASIC design, including synthesis of artificial, VLSI neural networks.

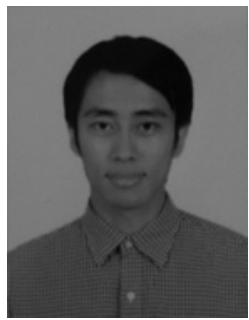

Dafeng Zhou received the B.Sc. degree in Microelectronics from Fudan University, Shanghai, China, in 2005, and the M.Sc. degree in Microelectronic Design from the University of Southampton, Southampton, UK in 2006. He is currently working toward the Ph.D. degree at the University of Southampton, Southampton, UK.

His current research interests include circuit-level modeling and simulation of carbon nanotube devices.

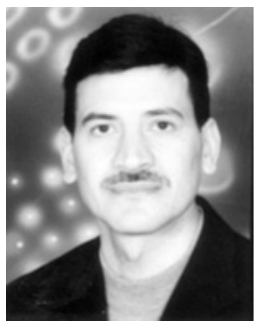

Bashir M. Al-Hashimi (M'99-SM'01-F'09) received the B.Sc. degree (with 1st-class classification) in Electrical and Electronics Engineering from the University of Bath, UK, in 1984 and the Ph.D. degree from York University, UK, in 1989. Following this he worked in the microelectronics design industry and in 1999, he joined the School of Electronics and Computer Science, Southampton University, UK, where he is currently a Full Professor of Computer Engineering and Director of the Pervasive System Center. He has authored one book on SPICE simulation, (CRC Press, 1995), and coauthored two books, Power Constrained Testing of VLSI circuits (Springer, 2002), and System-Level Design Techniques for Energy-Efficient Embedded Systems (Springer, 2004). In 2006, he edited the book, System-on-Chip: Next Generation Electronics (IEE Press, 2006). He has published over 240 papers in journals and refereed conference proceedings and supervised $25 \mathrm{PhD}$ theses successfully. His current research interests include low-power system-level design, system-onchip test, and reliable nano design.

Prof. Al-Hashimi is a Fellow of the IEE, and Fellow of the British Computer Society (BCS). He is the Editor-in-Chief of the IEE Proceedings: Computers and Digital Techniques, an editor of the Journal of Electronic Testing: Theory and Applications (JETTA), and is a member of the editorial board of the Journal of Low Power Electronics, and the Journal of Embedded Computing. He was the General Chair of the 11th IEEE European Test Symposium (UK 2006) and he is the Technical-Programme Chair of DATE 09 and General Chair 2011. He is the coauthor of the James Beausang Best Paper Award at the 2000 IEEE International Test Conference relating to low power BIST for RTL data paths, and a co-author of a paper on test data compression which has recently been selected for a Springer book featuring the most influential work over the 10 years of the Design Automation and Test in Europe (DATE) conference.

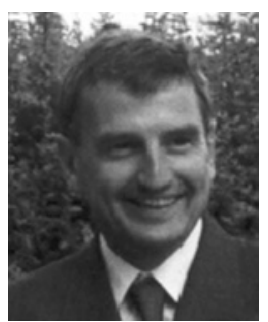

Peter Ashburn (M'82) was born in Rotherham, England in 1950. He received a B.Sc. degree in Electrical and Electronic Engineering in 1971 and a Ph.D. degree in 1974, both from the University of Leeds. His dissertation topic was an experimental and theoretical study of radiation damage in silicon p-n junctions.

In 1974, he joined the technical staff of Philips Research Laboratories and worked initially on ion implanted integrated circuit bipolar transistors, and then on electron lithography for sub-micron integrated circuits. In 1978 he joined the academic staff of the Department of Electronics and Computer Science of the University of Southampton as a lecturer, and currently is the holder of a Personal Chair in Nanoelectronics.

Since taking up a post at Southampton University, Professor Ashburn has worked on polysilicon emitter bipolar transistors, high-speed bipolar and BiCMOS technologies, gate delay expressions for bipolar circuits and the effects of fluorine in bipolar transistors. His current research interests include ultimate CMOS devices, carbon nanotube FETs and nanowire biosensors. $\mathrm{He}$ has authored and co-authored over 250 papers in the technical literature, given many invited papers and has authored books on the Design and Realization of Bipolar Transistors in 1988 and on Silicon Germanium Heterojunction Bipolar Transistors in 2003 